Reporte de caso

\title{
Fibroma desmoplásico de húmero. Reporte de caso y revisión de la literatura
}

\author{
Desmoplastic fibroma of humerus literature review, a case report
}

Juan Ramón Bonfil ${ }^{1}$ Juan Camilo Medina ${ }^{1,2}$ Alexis Eduardo Ponce ${ }^{1,2}$ Juan José Navarrete ${ }^{3}$ Pedro J

Delgado 4

${ }^{1}$ Hospital General de México, Módulo de Mano y Muñeca, Servicio de Ortopedia, México D.F. México.

${ }^{2}$ Universidad Nacional Autónoma de México, Facultad de Medicina, Especialización en Ortopedia y traumatología,

México D.F., México.

${ }^{3}$ Hospital General de México, Servicio de Patología, México D.F., México.

${ }^{4}$ Hospital Montepríncipe, Servicio de Ortopedia y Cirugía de Mano, Madrid, España.

\section{Información del artículo Resumen}

Recibido: 22/09/2020

Aceptado: 26/01/2021

Palabras clave: Fibroma desmoplásico, Húmero, Neoplasias óseas..

Keywords: Desmoplastic Fibroblatoma, Humerus, Bone Neoplasms.

DOI: https://doi.org/10.25214/28056272.1174
Introducción. El fibroma desmoplásico (FD) es un tumor óseo de características benignas, pero localmente agresivo, que se considera primario, raro, lítico y no metastásico; esta neoplasia representa el $0.1 \%$ de todos los tumores primarios de hueso. A continuación se presenta el caso de un paciente con FD en el cual el diagnóstico se estableció a través de los hallazgos radiológicos e histológicos y se describe la decisión de tratamiento.

Presentación del caso. Paciente masculino de 64 años, quien acudió al Servicio de Ortopedia del Hospital General de México por pérdida de la fuerza progresiva del brazo y antebrazo izquierdo con disminución de los rangos de movilidad y dolor en la zona. El sujeto fue diagnosticado con FD, intervenido con radioterapia por el servicio de radiooncología y manejado con una ortesis por el servicio de ortopedia. Se planteó la posibilidad de realizar transposición de los nervios mediano, cubital y radial, pero el paciente no aceptó el procedimiento.

\footnotetext{
Abstract

Introduction: Desmoplastic fibroma (DF) is a rare primary benign bone tumor, locally aggressive, lytic, but not metastatic. It has an incidence of $0.1 \%$ of all primary bone tumors. To present the diagnosis obtained through the radiological and histological findings and the treatment decision.

Case report: 64 -year-old male patient who in 2009 came to the clinic due to a loxosceles bite, 8 years later he was admitted again due to signs and symptoms in his left arm, which was diagnosed as FD.

Results: The patient is intervened by the radio-oncology service to start radiotherapy and managed with an orthosis by the Orthopedic service. Median, ulnar, and radial nerve transposition is proposed, but the patient does not accept the procedure.
} 


\section{Introducción}

El fibroma desmoplásico (FD) es un tumor óseo de características benignas, pero localmente agresivo, que se considera primario, lítico y no metastásico, y que representa el $0.1 \%$ de todos los tumores de hueso primario $^{1-3}$. Es importante mencionar que Fketcher et al. ${ }^{4}$ informaron, a partir de la clasificación de tumores de la Organización Mundial de la Salud (OMS), que a nivel histológico los FD se parecen a los tumores desmoides de tejido blando y producen abundante tejido de colágeno.

Los sitios donde los FD se presentan con mayor frecuencia son las metáfisis de los huesos largos (58\%), seguidos de la pelvis, el maxilar superior, el esternón y las vértebras ${ }^{2,5}$. Hasta el momento este tumor solo se ha reportado en tres informes en la diáfisis del húmero ${ }^{6-8}$.

Este tipo de neoplasias suelen ser más frecuentes en menores de 30 años y afectan en mayor proporción a los hombres $(2: 1)^{1,4,6,8,9}$. Las tasas de recaída se encuentran entre $37 \%$ y $72 \%$ posterior a la intervención quirúrgica ${ }^{10}$.

A continuación, se presenta el caso de un FD en un paciente que pertenece a una población en la que no es frecuente su ocurrencia, y además se informan los hallazgos clínicos, radiológicos e histológicos.

\section{Presentación del caso}

Hombre de 64 años con antecedente familiar de cáncer de estómago en su hermana y antecedentes personales de hipertensión arterial e hiperplasia prostática benigna en tratamiento médico, quien consultó al Servicio de Ortopedia del Hospital General de México por un cuadro clínico consistente en pérdida de la fuerza progresiva del brazo y antebrazo izquierdo con disminución de los rangos de movilidad acompañado de aumento de volumen, deformidad, inestabilidad y dolor. En la anamnesis, el paciente indicó que 8 años atrás, en 2009, acudió al servicio de urgencias de la misma institución por la picadura de una araña del género loxosceles en la cara posterior del brazo izquierdo, la cual le ocasionó edema masivo. De igual forma, el historial médico de esa fecha sugería un síndrome compartimental y contractura isquémica de volkmann ipsilateral, por lo que había sido manejado mediante fasciotomía palmar.

$\mathrm{Al}$ examen físico de ingreso se encontró que el brazo izquierdo presentaba aumento de volumen en tercio medio y distal con cambios de coloración de la piel (violácea) en región posterior y se evidenció una cicatriz en la cara anterior del tercio distal del húmero por toma de biopsia previa (Figuras 1 y 2 ). De igual forma, se estableció que el nervio mediano tenía una sensibilidad a 2 puntos $>6 \mathrm{~mm}$; el nervio cubital tenía una sensibilidad a 2 puntos de $1 \mathrm{~cm}$ con pérdida de la movilidad de los músculos intrínsecos de la mano y el aductor del pulgar, y el nervio radial no presentaba alteraciones y conservaba su sensibilidad. Los arcos de movilidad para la muñeca eran de $0^{\circ}$ a $40^{\circ}$, y de extensión, de $0^{\circ}$ a $20^{\circ}$ con fuerza muscular de $3 / 5$. También se registró incapacidad para la flexoextensión del codo.
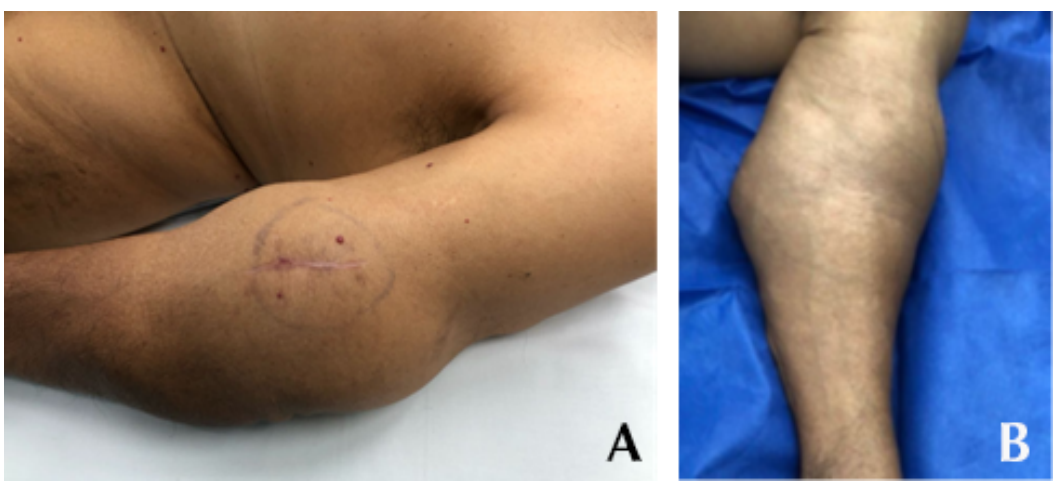

Figura 1. Visión lateral de fibroma desmoplásico de húmero izquierdo. A) visión anterior; B) aumento de volumen a nivel de codo izquierdo. Fuente: Documento obtenido durante la realización del estudio.

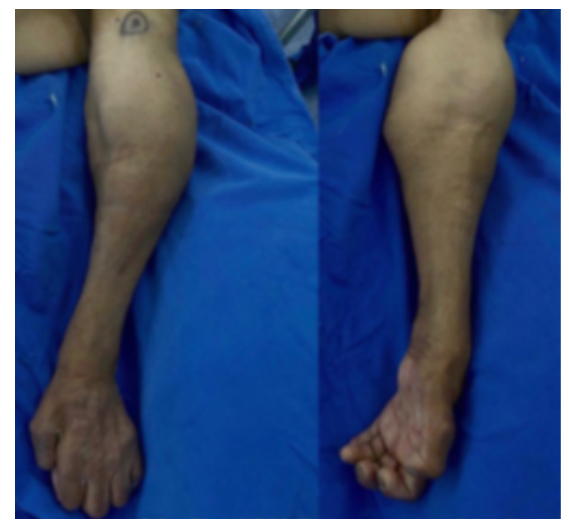

Figura 2. Visión anteroposterior de brazo izquierdo con fibroma desmoplásico, aumento de volumen a nivel de codo.

Fuente: Documento obtenido durante la realización del estudio. 
Las radiografías tomadas al momento del ingreso revelaron un aumento de volumen en los tejidos blandos del tercio distal del húmero y la destrucción ósea del húmero distal y el antebrazo proximal (Figura 3). Por su parte, la resonancia magnética nuclear (RMN) mostró una lesión hipointensa en T1 e hiperintensa en T2 de aspecto encapsulada con múltiples imágenes de aspecto condral en su interior y realce periférico tras la administración de medio de contraste; el reporte de este último paraclínico indicaba posible colección séptica secundaria a absceso con infiltración a hueso (Figura 4).
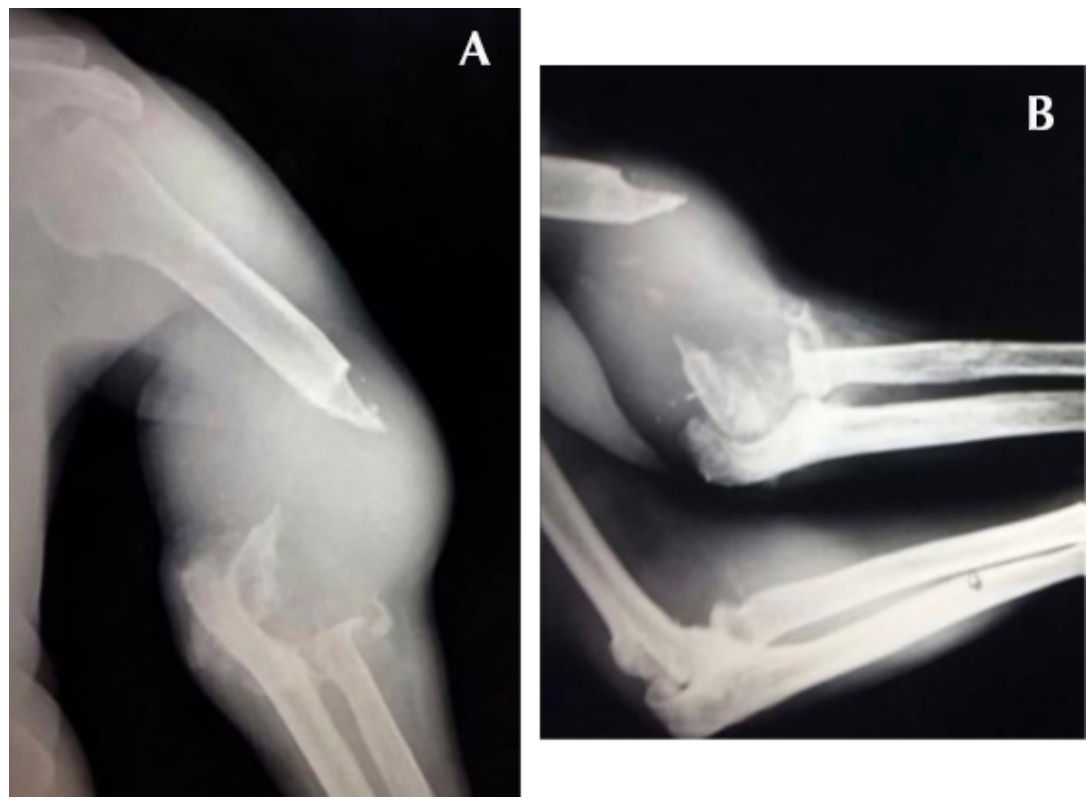

Figura 3. Radiografías simples con visión anterior (A) y lateral (B) de un brazo izquierdo con fibroma desmoplásico. Se evidencia destrucción ósea del húmero distal y antebrazo proximal.

Fuente: Documento obtenido durante la realización del estudio.
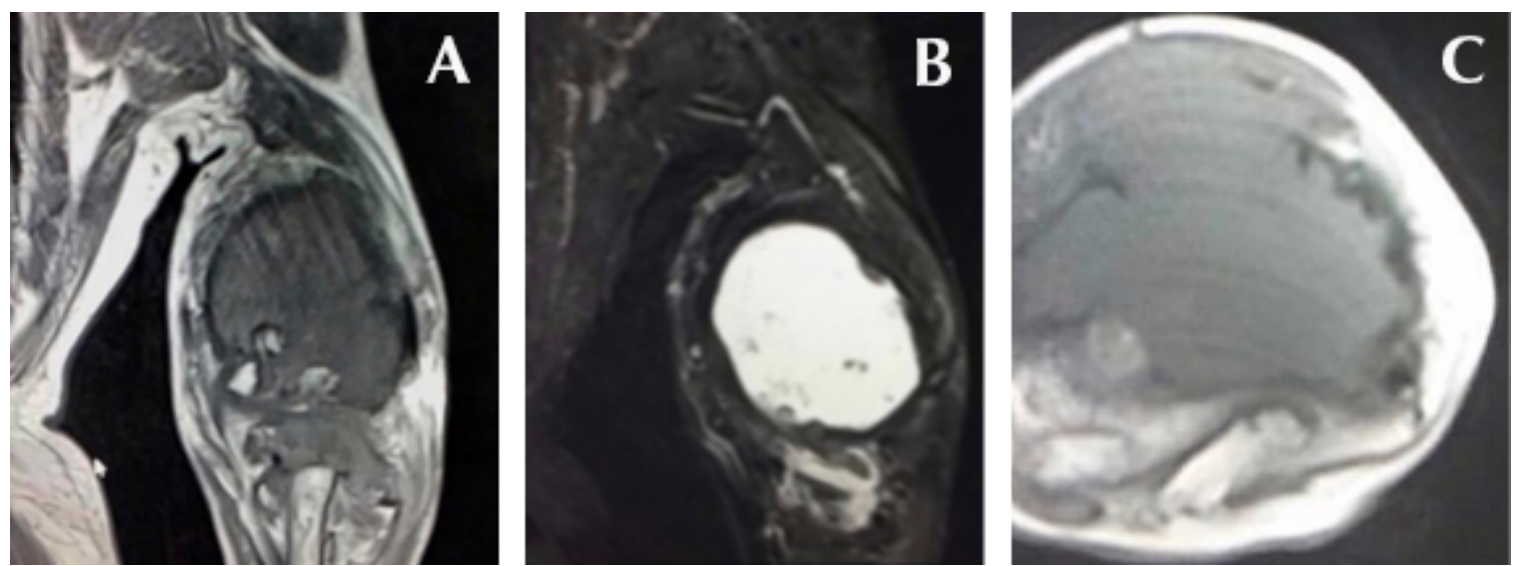

Figura 4. Resonancia magnética. A) corte coronal, T1 imagen hipointensa en tercio distal de húmero; B) corte coronal, T2 imagen hiperintensa de bordes bien definidos; C) corte axial, T1 tumoración hipointensa en tercio distal de brazo izquierdo con fibroma desmoplásico.

Fuente: Documento obtenido durante la realización del estudio.

Dados los hallazgos, inicialmente se plantearon como diagnósticos diferenciales condrosarcoma versus sarcoma sinovial versus colección séptica, por lo cual se completó el protocolo de estudio reali- zando biopsia incisional, pero esta no mostró ningún dato relevante. En segunda instancia se realizó una biopsia excisional que mostró un tejido fibroso que respaldaba el diagnóstico de FD (Figuras 5 y 6 ). 


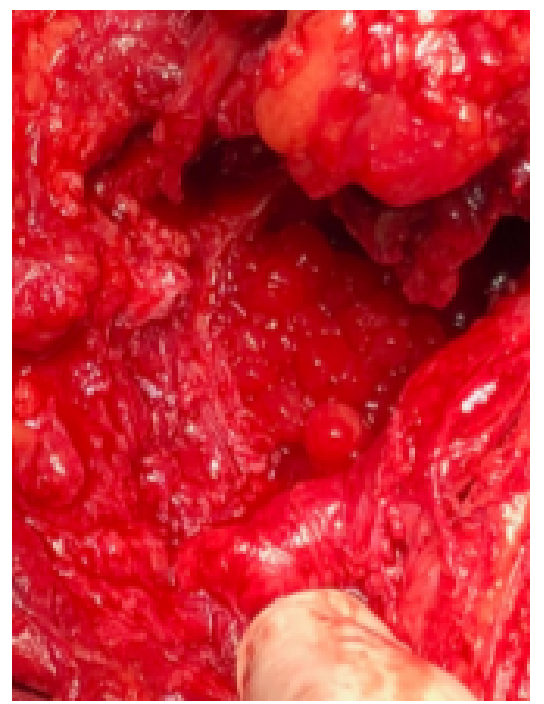

Figura 5. Imagen transquirúrgica donde se observan tumoraciones ovaladas múltiples.

Fuente: Documento obtenido durante la realización del estudio.

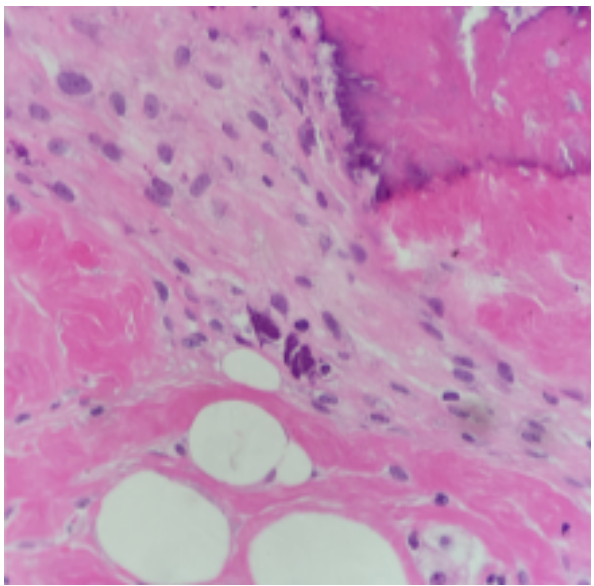

Figura 6. Corte histológico. En la microfotografía se observa el fibroma desmoplásico formado por células fusiformes que destruyen el hueso e infiltran tejido adiposo adyacente (H-E; 40x).

Fuente: Documento obtenido durante la realización del estudio.

Tras la confirmación del diagnóstico, se le explicó al paciente la rareza del tumor y se le indicó que las opciones de tratamiento eran remitirlo a interconsulta con oncología para que le realizaran radioterapia y con ortopedia para que le colocaran una ortesis. De igual forma, se le planteó la posibilidad de realizar una transposición de los nervios mediano, cubital y radial, pero el paciente no aceptó este procedimiento, ni tampoco la reconstrucción ósea con peroné libre.

\section{Discusión}

El FD es un tumor óseo raro y de características benignas ${ }^{4}$ que representa el $0.1-0.3 \%$ de todos los tumores óseos primarios ${ }^{10}$. Según la literatura consultada, estas neoplasias se han reportado en la pelvis, en la mandíbula, en la clavícula, en la columna, en el fémur, en la escápula, en el húmero y en la Tibia ${ }^{2,3,5-8,11-13}$. En el presente caso la lesión se ubicó en la región metafiso-diafisaria del húmero, que es un lugar poco común teniendo en cuenta que la literatura reporta que se presenta con mayor frecuencia en la región metafisaria ${ }^{6-8}$.

Respecto a la edad, se ha establecido que los FD se presentan con mayor frecuencia en adultos jóvenes, entre 30 y 40 años, pero en este caso el paciente no se encontraba en este rango de edad, aunque es importante mencionar que Okubo et al. ${ }^{11}$ ya había reportado el caso de una mujer de 65 años con esta condición.

Los signos y síntomas clínicos más frecuentes que pueden ocasionar los FD son: dolor, edema y disminución de arcos de movi$\operatorname{lidad}^{1,2,7}$, los cuales estuvieron presentes en el paciente reportado. Vale la pena mencionar que algunos pacientes con este tipo de tumores pueden ser asintomáticos.

Asimismo, es importante resaltar que los FD pueden confundirse en las imágenes diagnósticas con lesiones fibrosas benignas u otras más agresiva como los sarcomas. De acuerdo con lo anterior, la RMN presenta una baja intensidad ponderada por debajo de T2, lo cual, según la literatura, se atribuye a regiones de aumento del contenido de colágeno, mientras que otras áreas celulares son responsables de las regiones de mayor señal. En el presente caso, debido a los datos inespecíficos suministrados por la radiografía y la RMN, se decidió realizar una biopsia incisional, que tampoco fue concluyente.

Por otra parte, el nivel histológico de los FD es similar al de los tumores desmoides de tejidos blandos, en los cuales se presenta abundante producción de colágeno y muy poca actividad mitótica. ${ }^{1,8,12,13}$. De acuerdo con esto, dado que el reporte de la primera biopsia fue negativo para tumor, se decidió programar una biopsia excisional con la cual se pudo establecer el diagnóstico de FD.

\section{Conflicto de intereses}

Ninguno declarado por los autores.

\section{Financiación}

Ninguna declarada por los autores

\section{Agradecimientos}

Ninguno declarado por los autores.

\section{Referencias}

1. Ancillary E, Gross P. Desmoplastic Fibroma. En: Horvai A. High-Yield Pathology. Bone and Soft Tissue Pathology. 2012. p. 145-6.

2. Grube-Pagola P, Valle-Landa JC. Fibroma desmoplásico del fémur. Radiologia. 2013;55(4):359-61. DOI: 10.1016/j.rx.2011.12.003.

3. Kang DM, Juhng SK, Sohn YJ, Kim HS. Imaging findings of desmoplastic fibroma rarely involving the clavicle: case report. Korean J Radiol. 2014;15(1):130-3. DOI: 10.3348/kjr.2014.15.1.130.

4. Fletcher CDM, Unni KK, Mertens F. Pathology and Genetics of Tumours of Soft Tissue and Bone (World Health Organization Classification of Tumours). Oxford: Oxford University Press; 2002. 
5. Ma XN, Qiang S, Liu TY, Cao MY, Lv SC. Massive rare desmoplastic fibroma of the ilium and ischium in a young adult: a case report. Medicine (Baltimore). 2017;96(48):e8962. DOI: 10.1097/ MD.0000000000008962.

6. Apaydin M, Gelal F, Avci A, Adinas C, Sarsilmaz A, Varer M. Desmoplastic fibroma in humerus. J Med Imaging Radiat Oncol. 2008;52(5):489-90. DOI: 10.1111/j.1440-1673.2008.01989.x.

7. Aggarwal S, Gopinathan NR, Dhillon MS, Viswanathan VK. Diaphyseal desmoplastic fibroma of humerus - Report of a rare presentation. BMJ Case Rep. 2012;2012:bcr1020114954. DOI: 10.1136/bcr.10.2011.4954.

8. Böhm P, Kröber S, Greschniok A, Laniado M, Kaiserling E. Desmoplastic fibroma of the bone. A report of two patients, review of the literature, and therapeutic implications. Cancer. 1996;78(5):1011-23. DOI: 10.1002/(SICI)1097-0142(19960901) 78:5<1011::AID-CNCR11>3.0.CO;2-5.
9. $\quad$ Evans S, Ramasamy A, Jeys L, Grimer R. Desmoplastic fibroma of bone: A rare bone tumour. J Bone Oncol. 2014;3(3-4):77-9. DOI: 10.1016/j.jbo.2014.08.001.

10. Fletcher CDM, Bridge JA, Hogendoorn PCW, Mertens F, editores. WHO classification of tumours of soft tissue and bone. $4^{\text {th }}$ ed. International Agency for Research on Cancer; 2013.

11. Okubo T, Saito T, Takagi T, Torigoe T, Suehara Y, Akaike K, et al. Desmoplastic fibroma of the scapula with fluorodeoxyglucose uptake on positron emission tomography: A case report and literature review. Int J Clin Exp Pathol. 2013;6(10):2230-6.

12. Khatib B, Pogrel MA. Desmoplastic fibroma of the mandible in young children - a case series. Int J Oral Maxillofac Surg. 2017;46(2):173-80. DOI: 10.1016/j.ijom.2016.09.018.

13. Yin H, Zhang D, Wu Z, Yang X, Jiao J, Wan W, et al. Desmoplastic fibroma of the spine: A series of 12 cases and outcomes. Spine J. 2014;14(8):1622-8. DOI: 10.1016/j.spinee.2013.09.042. 